rheuma plus $2013 \cdot 12: 2$

DOI 10.1007/s12688-013-0081-5

○) Springer-Verlag Wien 2013

\author{
B. Leeb \\ Landesklinikum Weinviertel, Stockerau
}

\title{
Die Jahrestagung
}

Liebe Leserinnen und Leser, geht es Ihnen auch so? Die Zeit vergeht wie im Fluge. Haben wir gerade noch über einen Jahrhundertsommer geschwärmt oder geklagt, steht nun bereits der Jahreswechsel vor der Tür - mit ihm auch das für die Rheumatologen Österreichs wichtigste Fest des Jahres. Nein, Sie und ich wissen, dass nicht Weihnachten gemeint ist, sondern die Jahrestagung der ÖGR, die traditionellerweise Ende November stattfindet. Und zu diesem Ereignis erscheint auch die vierte Ausgabe von rheuma plus im Jahr 2013. Attila Dunky und ich hoffen, dass sowohl die ÖGR-Tagung als auch diese Ausgabe Ihre Aufmerksamkeit erregen und positive Anregungen bewirken können.

\section{Schwerpunkte auf der Jahrestagung}

Wie jedes Heft hat diese Ausgabe Ihren Fokus, der auch in der Programmgestaltung der ÖGR-Jahrestagung seinen Niederschlag findet. Die gemeinsame Sitzung der ÖGR mit der ÖGKM steht klar für die Bedeutung der Knochenkrankheiten in der Rheumatologie. Und dabei steht eine Erkrankung ganz besonders in Vordergrund der gemeinsamen Anstrengungen, nämlich die Osteoporose: Zwei Artikel befassen sich damit. Roland Kocijan vom $\mathrm{KH}$ der Barmherzigen Schwestern in Wien beschäftigt sich mit neuen therapeutischen Strategien zur Behandlung der Osteoporose. Dafür stehen ja seit längerem Bisphosphonate, selektive Östrogenrezeptor-Modulatoren, Strontium-Ranelat, Parathormon und auch ein monoklonaler Antikörper, Denosumab, zur Verfügung. Die Vorteile einer parenteralen Bisphosphonat-Therapie werden ebenso beleuchtet wie deren Kontraindikationen und unerwünschte Effekte, insbesonde- re die atypischen Frakturen. Auch ein kritischer Überblick über die neueren Substanzen wird gegeben. Als logische Konsequenz wird der Schluss gezogen, dass Bedarf nach therapeutischen Alternativen besteht, die einerseits wirksam, andererseits auch sicher und kostengünstig sein sollen.

Der Artikel von Bernhard Rintelen, dem Kassier und Leiter des Arbeitskreises Osteoporose der ÖGR, befasst sich mit der Glukokortikoid-induzierten Osteoporose, die klangvoll auch GIO abgekürzt wird; mehr schon ein Kosename, der aber die Bedeutung dieser Veränderung für die Betroffenen nicht verniedlichen soll. Vierzig bis fünfzig Prozent der Patienten mit RA werden nach wie vor mit Glukokortikoiden behandelt; selbstverständlich mit immer niedriger werdenden Dosierungen, aber eine Schwellendosis, ab der etwa Glukokortikoide unbedenklich zu geben wären, existiert nicht. Das heißt, wie Rintelen ausführt, mit dem Beginn einer Glukokortikoid-Therapie hat man an deren mögliche deletäre Wirkung auf den Knochen zu denken und entsprechend vorzugehen.

\section{Impfungen und Biologika}

Mit einem weiteren wesentlichen Thema der Prophylaxe bei entzündlich rheumatischen Erkrankungen befasst sich der Artikel von Hans-Peter Brezinschek aus Graz, nämlich mit den Impfungen. Nicht erst seit Einführung der Biologika-Therapie ist bekannt, dass Patienten mit RA ein im Vergleich zur Normalbevölkerung doppelt so hohes Risiko für Infektionen haben. Außerdem können Infekte bei diesen Patienten, besonders, wenn sie unter Biologika-Therapie stehen, einen schwereren Verlauf nehmen. Somit ergibt sich hier ein Berührungspunkt von Rheuma- tologie und Infektiologie mit höchster Bedeutung für die Patienten.

Stoff für Diskussion sollte diesmal auch die zwar etwas kürzere, aber trotzdem hoffentlich interessante Folge aus „BioReg berichtet“ liefern. Hier wird auf die Bedeutung der Tuberkuloseprophylaxe eingegangen, wie auch auf die Operationshäufigkeit unter Biologika-Therapie. Zum Nachdenken sollten vor allem die Zahlen der Sozialversicherung und die berichtete Inanspruchnahme von Physiound Ergotherapie anregen.

Zum Schluss möchten wir Sie, wie immer, herzlich dazu einladen, uns Beiträge zur gegenständlichen Ausgabe, aber auch zu allen rheumatologischen Themen, die Sie für relevant oder diskussionswert halten, zu schicken. Zum Abschluss möchte ich, wie immer, der Hoffnung Ausdruck geben, dass Sie in dieser Ausgabe für sich Interessantes finden werden und sage für Kritik, Hinweise, Zustimmung und für jeden anderen Beitrag schon im Voraus Danke!

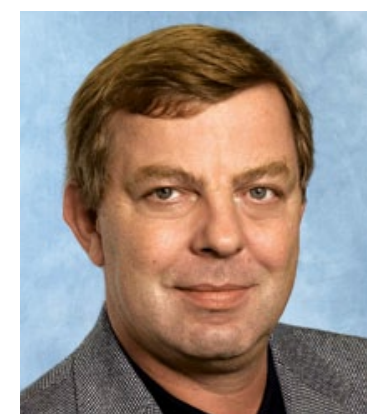

Herzlichst, Ihr

Burkhard Leeb 